International Mathematical Forum, 1, 2006, no. 33, 1645-1657

\title{
A buyer - seller game model for selection and negotiation of purchasing bids on interval data
}

\author{
G. R. Jahanshahloo, F. Hosseinzadeh Lotfi ${ }^{1}$, S. Razmian \\ Department of Mathematics, Science and Research Branch \\ Islamic Azad University, Tehran, Iran
}

\begin{abstract}
Selection and negotiation of purchasing bids is a complex decision making process that requires consideration of a variety of vendor attributes such as price, delivery performance, and quality. Several decision models have been utilized for vendor evaluation and selection. Talluri [EUR. J. Operat. Res. 143(1) (2002) 171] and Zhu [EUR. J. Operat. Res. 154(2004) 150] propose a buyer - seller game model that evaluate the efficiency of alternative bids with respect to the ideal target set by the buyer. We prove some theorem in the current paper about this model. The bids cannot be easily evaluated and selected, while inputs and outputs each vary in interval. In this paper, presenting a new idea for evaluating the bids with interval data, an interval will be defined for the efficiency score of each bid. And finally, a method for selecting bids by the obtained efficiency interval is presented. And, the new technique will be applied to a set of real data.
\end{abstract}

Mathematics Subject Classification: Operations Research, 90.

Keywords: Game model, Integer programming, Linear programming, Efficiency, Data envelopment analysis, Interval data.

\section{Introduction}

As pointed out by Wise and Morrison (2000), one of the major flaws in the current business - to - business (B2B) model is that it focuses on price - driven transactions between buyers and sellers. In fact, a number of efficiency - based on negotiation models have been developed to deal with multiple attributes inputs and outputs. For example, data envelopment analysis (DEA) is used

\footnotetext{
${ }^{1}$ Corresponding author, Tel: +98 - 21- 4804172, Fax: +98 - 21 - 4804172, P. O. Box: 14155/775 and 14155/4933, Post code: 1477893855.
} 
by Weber and Desai (1996) and Weber et al. (1998) to develop models for vendor evaluation and negotiation. Vijayan (2000) discusses the importance of considering multiple vendor related attributes. It is suggested by him that companies are developing software that is considering several factors beyond price for effective $\mathrm{B} 2 \mathrm{~B}$ transactions.

Based on a survey of 170 purchasing managers, Dickson (1966) suggested that cost, quality, and delivery performance are the three most important criteria in vendor evaluatoin.

Willis et al. (1993) proposed a classification of vendor performance evaluation models that included categorical, weighted points, and cost ratio approaches.

Weber and Desai (1996) and Weber et al. (1998) identified two articles about decision models for vendor evaluation and negotiation bids.

In this paper, we review the models of Talluri (2002) and Zhu (2004) and prove some theorem.

In the recent years, models are observed to have inputs and outputs as interval. In this paper, an interval will be defined for each bid's efficiency score, and a method for selecting bids by the obtained efficiencie's interval is presented.

This paper consists of the following sections: In section 2, the models in Talluri (2002) and Zhu (2004) are discussed and some theorems are proved. In

section 3, this model with interval data is presented. One example with real interval data is provided. Finally, the conclusion is given.

\section{Models introduce}

In this section, we review the models of Talluri (2002) and Zhu (2004). Since the evaluations in these models are conducted from a buyer's perspective, inputs are defined as the resources spent and outputs as the benefits derived by the buyer. The scenario include single input - single output, single input multiple output and multiple input - multiple output cases.

Case1 (single input - single output) Expression (1) depicts the model.

$$
e_{k}=\frac{\frac{y_{k}}{x_{k}}}{\max \left\{\frac{y_{j}}{x_{j}}: j=1, \ldots, n\right\}}
$$

Then, when we have single input - single output, there is no require of the weights.

Case2 (single input - multiple output with simultaneous consideration 
of $n$ vendor bids) The model of Zhu (2004) is shown in (2).

$$
\begin{array}{lll}
\max & \sum_{j=1}^{n}\left(\frac{\sum_{r=1}^{s} a_{r} y_{r j}}{b_{1} x_{j}}\right) \\
\text { s.t. } & \frac{\sum_{r=1}^{s} a_{r} y_{r I}}{b_{1} x_{I}}=1, & \\
& \frac{\sum_{r=1}^{s} a_{r} y_{r j}}{b_{1} x_{j}} \leq 1, & j=1, \ldots, n, \\
& a_{r}, b_{1} \geq 0 & r=1, \ldots, s .
\end{array}
$$

Where $n$ represents the number of bids. $a_{r}, b_{1}$ represent unknown output and input weights, respectively. Each bid $j$ has $s$ outputs $y_{r j}(r=1, \ldots, s)$, $y_{r I}(r=1, \ldots, s)$ and $x_{I}$ represent the best value for the $r$-th output and the best value for the input.

Because there is one input, so we can consider $b_{1}=1$. Since $y_{r j}$ and $x_{j}$ are scalar, this form is linear. Model (2) identifies the vendor who has the maximum efficiency with respect to the ideal target. If we regard this model with minimization form [Talluri (2002)], the buyer can determine the largest minimum efficiency, when the efficiency of target is assumed to be one. Let $h_{p}^{*}$ and $\overline{h_{p}^{*}}$ be the optimal values to this models, and $h_{p}$ be the true efficiency of vendor $\mathrm{p}$ with respect to the fact that the efficiencies of the targets are sets equal to one, then $h_{p}^{*} \leq h_{p} \leq \overline{h_{p}^{*}}$. When both models are calculated, the buyer can have an efficiency range which the true efficiency lying within. This helps the vendor better in evaluating and selecting the vendors.

Case3 (single input - multiple output with individual consideration of $n$ vendor bids) Expression (3) depicts the model.

$$
\begin{aligned}
& \max \quad \frac{\sum_{r=1}^{s} a_{r} y_{r p}}{x_{p}} \\
& \text { s.t. } \quad \frac{\sum_{r=1}^{s} a_{r} y_{r I}}{x_{I}}=1 \text {, } \\
& \frac{\sum_{r=1}^{s} a_{r} y_{r j}}{x_{j}} \leq 1, \quad j=1, \ldots, n, \\
& a_{r} \geq 0 \quad r=1, \ldots, s .
\end{aligned}
$$

Where $p$ represents the vendor being evaluated. This model is a linear programming.

Case 4 (multiple input - multiple output with simultaneous consideration 
of $n$ vendor bids) Expression (4) depicts the model.

$$
\begin{aligned}
& \max \quad \sum_{j=1}^{n}\left(\frac{\sum_{r=1}^{s} a_{r} y_{r j}}{\sum_{i=1}^{m} b_{i} x_{i j}}\right) \\
& \text { s.t. } \quad \frac{\sum_{r=1}^{s} a_{r} y_{r I}}{\sum_{i=1}^{m} b_{i} x_{i I}}=1 \text {, } \\
& \frac{\sum_{r=1}^{s} a_{r} y_{r j}}{\sum_{i=1}^{m} b_{i} x_{i j}} \leq 1, \quad j=1, \ldots, n, \\
& a_{r}, b_{i} \geq 0 \quad r=1, \ldots, s, i=1, \ldots, m .
\end{aligned}
$$

Where $m$ represents the number of bid's inputs and $b_{i}$ represent unknown input weights. This model is non - linear programming. Talluri (2002) states that" the objective function can be replaced by $\frac{\sum_{r=1}^{s} a_{r}\left(\sum_{j=1}^{n} \frac{y_{r j}}{n}\right)}{\sum_{i=1}^{m} b_{i}\left(\sum_{j=1}^{n} \frac{x_{j j}}{n}\right)}$ ", but the objective function isn't equal to this expression, and this model isn't converted to a linear program.

Case 5 (multiple input - multiple output with individual consideration of $n$ vendor bids)

Expression (5) depicts the model.

$$
\begin{aligned}
& \max \quad \frac{\sum_{r=1}^{s} a_{r} y_{r p}}{\sum_{i=1}^{m} b_{i} x_{i p}} \\
& \text { s.t. } \quad \frac{\sum_{r=1}^{s} a_{r} y_{r I}}{\sum_{i=1}^{m} b_{i} x_{i I}}=1, \\
& \frac{\sum_{r=1}^{s} a_{r} y_{r j}}{\sum_{i=1}^{m} b_{i} x_{i j}} \leq 1, \quad j=1, \ldots, n, \\
& a_{r}, b_{i} \geq 0 \quad r=1, \ldots, s, i=1, \ldots, m .
\end{aligned}
$$

This model by replacing the objective function's denominator with $\frac{1}{t}$ is transformed to a linear form.

Theorem 2.1 Optimal value of objective function in (4) is less than or equal to objective function's optimal value in (5).

Proof: Assume $(\bar{a}, \bar{b})$ to be optimal solution for objective function in $(4)$, thus: $e_{p}=\frac{\bar{a} y_{p}}{\bar{b} x_{p}}$. obviously $(\bar{a}, \bar{b})$ is a feasible solution for $(5)$. Then the optimal solution of (4) is less than or equal to optimal solution of (5). 


\subsection{Integer programming model for bid selection}

The vendor ratings are evaluated in a 0 - 1 integer programming model, shown in expression (5).

$$
\begin{aligned}
& \min \quad z_{0}=\sum_{j=1}^{n} z_{j} \\
& \text { s.t. } \quad \sum_{j=1}^{n} \theta_{j} z_{j}-\theta_{\text {avg }} \sum_{j=1}^{n} z_{j} \geq 0 \text {, } \\
& \sum_{j=1}^{n} q_{j}=D \text {, } \\
& q_{j}-c_{\text {jmax }} z_{j} \leq 0, \quad j=1, \ldots, n, \\
& q_{j}-o_{j \min } z_{j} \geq 0, \quad j=1, \ldots, n, \\
& q_{j} \geq 0 \quad j=1, \ldots, n, \\
& z_{j} \in\{0,1\} \quad j=1, \ldots, n .
\end{aligned}
$$

Where $z_{j}$ is the binary variable that represents the selection status of vendor bid $j, \theta_{j}$ is the efficiency of vendor bid $\mathrm{j}, \theta_{\text {avg }}$ is average efficiency target, $q_{j}$ is the amount ordered from vendor $j, D$ is the buyer's demand requirement, $c_{j \max }$ is the capacity of vendor $j, o_{j m i n}$ is the minimum order quantity requirement of vendor $j$.

Theorem $2.2 z_{0}>0$ for all feasible solution in (6).

Proof: Since $z_{j} \in\{0,1\}$ for every $j$, thus $z_{0}>0$ or $z_{0}=0$. Let $z_{0}=0$, then $z_{j}=0$ for every $j$. From $q_{j}-o_{j m i n} z_{j} \geq 0$ we have $q_{j} \geq 0$, and from $q_{j}-c_{j \max } z_{j} \leq 0$, we have $q_{j} \leq 0$, therefore $q_{j}=0$, that contradicts $\sum_{j=1}^{n} q_{j}=D$, because $D$ is become zero, therefore $z_{0}>0$.

Theorem 2.3 Let in bids we have at least one nonideal vendor, then in (5) the vendor that has efficiency score 1 , in (6) related $z_{j}$ is 1.

Proof: Since there exist $D M U_{t}$ subject to $\theta_{t}<1$, so $\sum_{j=1, j \neq p}^{n}\left(1-\theta_{j}\right)>0$, therefore

$n-1=\sum_{j=1, j \neq p}^{n} 1>\sum_{j=1, j \neq p}^{n} \theta_{j}$, we have

$n-1>(n-n+1) \sum_{j=1, j \neq p}^{n} \theta_{j}=n \sum_{j=1, j \neq p}^{n} \theta_{j}-(n-1) \sum_{j=1, j \neq p}^{n} \theta_{j}$, we conclude $\frac{\sum_{j=1, j \neq p}^{n} \theta_{j}}{n-1}<\frac{\sum_{j=1, j \neq p}^{n} \theta_{j}+1}{n}=\frac{\sum_{j=1, j \neq p}^{n} \theta_{j}+\theta_{p}}{n}=\frac{\sum_{j=1}^{n} \theta_{j}}{n}=\theta_{\text {avg }}$. Now assume to the contrary that $\theta_{p}=1$ and $z_{p}=0$, then:

$$
\begin{aligned}
& 0 \leq \sum_{j=1}^{n} \theta_{j} z_{j}-\theta_{\text {avg }} \sum_{j=1}^{n} z_{j}=\sum_{j=1, j \neq p}^{n} \theta_{j} z_{j}-\theta_{\text {avg }} \sum_{j=1, j \neq p}^{n} z_{j} \\
& \leq \sum_{j=1, j \neq p}^{n} \theta_{j}-(n-1) \theta_{\text {avg }}=(n-1)\left(\frac{\sum_{j=1, j \neq p}^{n} \theta_{j}}{n-1}-\theta_{\text {avg }}\right)<0 .
\end{aligned}
$$

This contradictions complete the proof, and if $\theta_{p}=1$ then certainly $z_{p}=1$. 


\section{$3 \quad$ Buyer - seller game model with interval data} is:

Let input and output values of any bid be located in a certain interval, that

$$
\forall i, \forall j \quad x_{i j} \in\left[x_{i j}^{l}, x_{i j}^{u}\right] \quad \text { and } \quad \forall r, \forall j \quad y_{r j} \in\left[y_{r j}^{l}, y_{r j}^{u}\right]
$$

Therefore relative efficiency of $D M U_{p}$ is also located in an interval. Now we evaluate the identified models for interval data.

\subsection{Case (3) with interval data}

The following pair of LP models has been developed to generate the upper and lower bounds of interval efficiency for each vendor.

$$
\begin{aligned}
& e_{p}^{l}=\max \quad \sum_{r=1}^{s} a_{r} \frac{y_{r p}^{l}}{x_{p}^{u}} \\
& e_{p}^{u}=\max \quad \sum_{r=1}^{s} a_{r} \frac{y_{r p}^{u}}{x_{p}^{l}} \\
& \text { s.t. } \quad \sum_{r=1}^{s} a_{r} \frac{y_{r I}^{u}}{x_{I}^{l}}=1, \\
& \text { s.t. } \quad \sum_{r=1}^{s} a_{r} \frac{y_{r I}^{l}}{x_{I}^{u}}=1, \\
& \sum_{r=1}^{s} a_{r} \frac{y_{r j}^{u}}{x_{j}^{l}} \leq 1, \quad j \neq p, \\
& \sum_{r=1}^{s} a_{r} \frac{y_{r j}^{l}}{x_{j}^{u}} \leq 1, \quad j \neq p, \\
& \sum_{r=1}^{s} a_{r} \frac{y_{r p}^{l}}{x_{p}^{u}} \leq 1, \\
& \sum_{r=1}^{s} a_{r} \frac{y_{r p}^{u}}{x_{p}^{l}} \leq 1, \\
& a_{r} \geq 0 \quad r=1, \ldots, s . \\
& a_{r} \geq 0 \quad r=1, \ldots, s .
\end{aligned}
$$

The dual of models (3) and (7) and (8) are as follows, respectively:

$$
\begin{aligned}
& e_{p}=\min \quad \theta+\sum_{j=1, j \neq p}^{n} \lambda_{j}+\lambda_{p} \\
& \text { s.t. } \quad \sum_{j=1, j \neq p}^{n} \lambda_{j}\left(\frac{y_{r j}}{x_{j}}\right)+\lambda_{p}\left(\frac{y_{r p}}{x_{p}}\right)+\theta\left(\frac{y_{r I}}{x_{I}}\right) \geq \frac{y_{r p}}{x_{p}}, \quad r=1, \ldots, s \\
& \lambda_{j} \geq 0 \quad j=1, \ldots, n, \\
& \theta \quad \text { free. } \\
& e_{p}^{l}=\min \quad \theta^{\prime}+\sum_{j=1, j \neq p}^{n} \lambda_{j}^{\prime}+\lambda_{p}^{\prime} \\
& \text { s.t. } \quad \sum_{j=1, j \neq p}^{n} \lambda_{j}^{\prime}\left(\frac{y_{r j}^{u}}{x_{j}^{l}}\right)+\lambda_{p}^{\prime}\left(\frac{y_{r p}^{l}}{x_{p}^{u}}\right)+\theta^{\prime}\left(\frac{y_{r I}^{u}}{x_{I}^{l}}\right) \geq \frac{y_{r p}^{l}}{x_{p}^{u}}, \quad r=1, \ldots, s, \\
& \lambda_{j}^{\prime} \geq 0 \\
& j=1, \ldots, n, \\
& \theta^{\prime} \quad \text { free. } \\
& e_{p}^{u}=\min \quad \theta^{\prime \prime}+\sum_{j=1, j \neq p}^{n} \lambda_{j}^{\prime \prime}+\lambda_{p}^{\prime \prime} \\
& \text { s.t. } \quad \sum_{j=1, j \neq p}^{n} \lambda_{j}^{\prime \prime}\left(\frac{y_{r j}^{l}}{x_{j}^{u}}\right)+\lambda_{p}^{\prime \prime}\left(\frac{y_{r p}^{u}}{x_{p}^{l}}\right)+\theta^{\prime \prime}\left(\frac{y_{r I}^{l}}{x_{I}^{u}}\right) \geq \frac{y_{r p}^{u}}{x_{p}^{l}}, r=1, \ldots, s, \\
& \lambda_{j}^{\prime \prime} \geq 0 \\
& j=1, \ldots, n, \\
& \theta^{\prime \prime} \quad \text { free. }
\end{aligned}
$$


Theorem 3.1 Let $\left(\theta, \lambda_{1}, \ldots, \lambda_{n}\right)$ be an optimal solution to model (9), then $\theta \geq 0$.

Proof: In model (3) the ideal constraint can be expressed as $\frac{\sum_{r=1}^{s} a_{r} y_{r I}}{x_{I}} \leq 1$, because in optimal solution we always have: $\frac{\sum_{r=1}^{s} a_{r} y_{r I}}{x_{I}}=1$. Since, let $a^{*}$ be an optimal solution, but we have $\sum_{r=1}^{s} \frac{a_{r}^{*} y_{r I}}{x_{I}}<1$. I know there exists $t$ such that $\sum_{r=1}^{s} \frac{a_{r}^{*} y_{r t}}{x_{t}}=1$, if $t=i$ the theorem is proved, othere wise since $x_{I} \leq x_{t}$ and $y_{r I} \geq y_{r t}$, forall $\mathrm{r}$ then: $x_{I} \leq x_{t}$ and $a_{r}^{*} y_{r I} \geq a_{r}^{*} y_{r t}$, forall $\mathrm{r}$. Hence $1>\sum_{r=1}^{s} \frac{a_{r}^{*} y_{r I}}{x_{I}} \geq \sum_{r=1}^{s} \frac{a_{r}^{*} y_{r t}}{x_{t}}=1$, where it is a contradiction. Thus in optimal solution, we can write the ideal constraint with equality form. Therefore in optimal solution $\theta$ is non-negative.

Theorem 3.2 Let $\left(\theta^{*}, \lambda^{*}\right)$ be an optimal solution to model (9), then $\left(1-\lambda_{p}^{*}\right) \geq$ 0 .

Proof: Consider the following model

$$
\begin{array}{cl}
\min & \sum_{j=1}^{n} \lambda_{j} \\
\text { s.t. } & \sum_{j=1}^{n} \lambda_{j}\left(\frac{y_{r j}}{x_{j}}\right) \geq \frac{y_{r p}}{x_{p}}, \\
\lambda \geq 0 & \quad r=1, \ldots, s,
\end{array}
$$

$\lambda=e_{p}$, is a feasible solution. Then the optimal objective function's value is always less than or equal to unity, thus $\lambda_{j}^{*} \leq 1$, for every $j$. Since if there exists $j$ such that $\lambda_{j}^{*}>1$, from $\sum_{j=1}^{n} \lambda_{j}^{*} \leq 1$, it follows that there exist at least one $t$ such that $\lambda_{t}<0$, this contradicts $\lambda \geq 0$. So $\lambda_{j} \leq 1$ for every $j$, especially for $j=p$. There fore $\left(1-\lambda_{p}^{*}\right) \geq 0$.

Theorem 3.3 In models (3) and (7) and (8) we always have: $e_{p}^{l} \leq e_{p} \leq e_{p}^{u}$.

Proof: Suppose that $(\theta, \lambda)$ is the optimal solution to model $(9)$, with respect to

$y_{r j}^{u} \geq y_{r j} \geq y_{r j}^{l}$ for every $r$ and $x_{j}^{l} \leq x_{j} \leq x_{j}^{u}$ and by theorem (4) and (5) we have:

$\sum_{j=1, j \neq p}^{n} \lambda_{j}\left(\frac{y_{r j}^{u}}{x_{j}^{l}}\right)+\theta\left(\frac{y_{r I}^{u}}{x_{I}^{l}}\right) \geq \sum_{j=1, j \neq p}^{n} \lambda_{j}\left(\frac{y_{r j}}{x_{j}}\right)+\theta\left(\frac{y_{r I}}{x_{I}}\right) \geq\left(1-\lambda_{p}\right) \frac{y_{r p}}{x_{p}} \geq\left(1-\lambda_{p}\right) \frac{y_{r p}^{l}}{x_{p}^{u}}$

Which means $(\theta, \lambda)$ is also a feasible solution to model (10), so we get $e_{p}^{l} \leq e_{p}$.

Now, if $\left(\theta^{\prime \prime}, \lambda^{\prime \prime}\right)$ is the optimal solution to model (11) similary, is proved that $\theta^{\prime \prime} \geq 0$ and $\left(1-\lambda_{p}^{\prime \prime}\right) \geq 0$. Then the following inequalities hold:

$\sum_{j=1, j \neq p}^{n} \lambda_{j}^{\prime \prime}\left(\frac{y_{r j}}{x_{j}}\right)+\theta^{\prime \prime}\left(\frac{y_{r I}}{x_{I}}\right) \geq \sum_{j=1, j \neq p}^{n} \lambda_{j}^{\prime \prime}\left(\frac{y_{r j}^{l}}{x_{j}^{u}}\right)+\theta^{\prime \prime}\left(\frac{y_{r I}^{l}}{x_{I}^{u}}\right) \geq\left(1-\lambda_{p}^{\prime \prime}\right) \frac{y_{r p}^{u}}{x_{p}^{l}} \geq\left(1-\lambda_{p}^{\prime \prime}\right) \frac{y_{r p}}{x_{p}}$

It is obvious that $\left(\theta^{\prime \prime}, \lambda^{\prime \prime}\right)$ is a feasible solution of model (9). Thus, we have the inequality relation: $e_{p} \leq e_{p}^{u}$. Therefore $e_{p}^{l} \leq e_{p} \leq e_{p}^{u}$ and complete the proof. 


\subsection{Case (5) with interval data}

The upper and lower bounds of the relative efficiency of $D M U_{p}$ in (5) are obtained by solving the following problems:

$$
\begin{aligned}
& e_{p}^{l}=\max \frac{\sum_{r=1}^{s} a_{r} y_{r p}^{l}}{\sum_{i=1}^{m} b_{i} x_{i p}^{u}} \\
& e_{p}^{u}=\max \frac{\sum_{r=1}^{s} a_{r} y_{r p}^{u}}{\sum_{i=1}^{m} b_{i} x_{i p}^{l}} \\
& \text { s.t. } \quad \frac{\sum_{r=1}^{s} a_{r} y_{r I}^{u}}{\sum_{i=1}^{m} b_{i} x_{i I}^{l}}=1, \\
& \text { s.t. } \quad \frac{\sum_{r=1}^{s} a_{r} y_{r I}^{l}}{\sum_{i=1}^{m} b_{i} x_{i I}^{u}}=1, \\
& \frac{\sum_{r=1}^{s} a_{r} y_{r j}^{u}}{\sum_{i=1}^{m} b_{i} x_{i j}^{l}} \leq 1, \quad j \neq p, \\
& \frac{\sum_{r=1}^{s} a_{r} y_{r j}^{l}}{\sum_{i=1}^{m} b_{i} x_{i j}^{u}} \leq 1, \quad j \neq p, \\
& \frac{\sum_{r=1}^{s} a_{r} y_{r p}^{l}}{\sum_{i=1}^{m} b_{i} x_{i p}^{u}} \leq 1, \\
& \frac{\sum_{r=1}^{s} a_{r} y_{r p}^{u}}{\sum_{i=1}^{m} b_{i} x_{i p}^{l}} \leq 1 \text {, } \\
& a_{r} \geq 0 \quad r=1, \ldots s, \\
& a_{r} \geq 0 \quad r=1, \ldots s, \\
& b_{i} \geq 0 \quad i=1, \ldots, m . \\
& b_{i} \geq 0 \quad i=1, \ldots, m \text {. }
\end{aligned}
$$

Consider dual of models:

$e_{p}=\min \quad \theta$

s.t.

$$
\begin{array}{cc}
\mu y_{r I}+\sum_{j=1, j \neq p}^{n} \lambda_{j} y_{r j}+\lambda_{p} y_{r p} \geq y_{r p}, & r=1, \ldots, s, \\
\theta x_{i p}-\mu x_{i I}-\sum_{j=1, j \neq p}^{n} \lambda_{j} x_{i j}-\lambda_{p} x_{i p} \geq 0, & i=1, \ldots, m, \\
\lambda_{j} \geq 0 & j=1, \ldots, n, \\
\theta, \mu \quad \text { free. } &
\end{array}
$$

$e_{p}^{l}=\min \quad \theta^{\prime}$

$$
\text { s.t. } \quad \mu^{\prime} y_{r I}^{u}+\sum_{j=1, j \neq p}^{n} \lambda_{j}^{\prime} y_{r j}^{u}+\lambda_{p}^{\prime} y_{r p}^{l} \geq y_{r p}^{l}, \quad r=1, \ldots, s,
$$

$$
\begin{array}{cc}
\theta^{\prime} x_{i p}^{u}-\mu^{\prime} x_{i I}^{l}-\sum_{j=1, j \neq p}^{n} \lambda_{j}^{\prime} x_{i j}^{l}-\lambda_{p}^{\prime} x_{i p}^{u} \geq 0, & i=1, \ldots, m, \\
\lambda_{j}^{\prime} \geq 0 & j=1, \ldots, n, \\
\theta^{\prime}, \mu^{\prime} \quad \text { free. } &
\end{array}
$$

$e_{p}^{u}=\min \quad \theta^{\prime \prime}$

$$
\begin{array}{ll}
\text { s.t. } & r=1, \ldots, s, \\
\mu^{\prime \prime} y_{r I}^{l}+\sum_{j=1, j \neq p}^{n} \lambda_{j}^{\prime \prime} y_{r j}^{l}+\lambda_{p}^{\prime \prime} y_{r p}^{u} \geq y_{r p}^{u}, & i=1, \ldots, m, \\
\theta^{\prime \prime} x_{i p}^{l}-\mu^{\prime \prime} x_{i I}^{u}-\sum_{j=1, j \neq p}^{n} \lambda_{j}^{\prime \prime} x_{i j}^{u}-\lambda_{p}^{\prime \prime} x_{i p}^{l} \geq 0, & j=1, \ldots, n, \\
\lambda_{j}^{\prime \prime} \geq 0 & \\
\theta^{\prime \prime}, \mu^{\prime \prime} \quad \text { free. } &
\end{array}
$$

Theorem 3.4 If $(\theta, \lambda, \mu)$ be an optimal solution of model (14), then $\mu \geq 0$. 
Proof: Consider linear form of (5), since in optimal solution we have: $\sum_{r=1}^{s} a_{r}^{*} y_{r I}-\sum_{i=1}^{m} b_{i}^{*} x_{i I}=0$, the linear form can be written as follows:

$$
\begin{array}{rlr}
e_{p}=\max & \sum_{r=1}^{s} a_{r} y_{r p} \\
\text { s.t. } & \sum_{r=1}^{s} a_{r} y_{r I}-\sum_{i=1}^{m} b_{i} x_{i I} \leq 0, & (17) \\
& \sum_{r=1}^{s} a_{r} y_{r j}-\sum_{i=1}^{m} b_{i} x_{i j} \leq 0, & j=1, \ldots, n, \\
& \sum_{i=1}^{m} b_{i} x_{i p}=1, & \\
& a_{r}, b_{i} \geq 0 & r=1, \ldots, s, i=1, \ldots, m .
\end{array}
$$

Suppose to the contrary that $\left(a^{*}, b^{*}\right)$ be an optimal solution such that satisfies $a^{*} y_{I}-b^{*} x_{I}<0$, but we know that there exist $t$ such that $a^{*} y_{t}-b^{*} x_{t}=0$, if $t=I$ the proof is completed. Othere wise since $y_{I} \geq y_{t}$ and $x_{I} \leq x_{t}$ then $b^{*} x_{I} \leq b^{*} x_{t}$ and $a^{*} y_{I} \geq a^{*} y_{t}$. It follows that $0>a^{*} y_{I}-b^{*} x_{I} \geq a^{*} y_{t}-b^{*} x_{t}$ where this is a contradiction. Hence ideal constraint's form in optimal solution is equality, then $\mu \geq 0$.

Theorem 3.5 If $(\theta, \lambda, \mu)$ be an optimal solution of (14), then $\left(\theta-\lambda_{p}\right) \geq 0$.

Proof: Since $X_{p} \neq 0$ there exist $t$ such that $x_{t p}>0$. Now consider

$$
\mu x_{t I}+\sum_{j=1, j \neq p}^{n} \lambda_{j} x_{t j} \leq\left(\theta-\lambda_{p}\right) x_{t p}
$$

Suppose to the contrary that $\left(\theta-\lambda_{p}\right)<0$, then $\mu x_{t I}+\sum_{j=1, j \neq p}^{n} \lambda_{j} x_{t j}<0$. This means that we must have $\mu x_{t I}<0$ or there exist $l$ such that $\lambda_{l} x_{t l}<0$, that with respect to $\mu \geq 0$ and $X \geq 0$ and $\lambda \geq 0$ this is contradiction. There fore $\left(\theta-\lambda_{p}\right) \geq 0$.

Theorem 3.6 In models (5) and (12) and (13) we always have: $e_{p}^{l} \leq e_{p} \leq e_{p}^{u}$.

Proof: Assume that $(\theta, \lambda, \mu)$ be an optimal solution of model (14), it is proved as same as the theorem (5) that $0 \leq \lambda_{p} \leq 1$, by theorem (7) and (8) we have:

$\mu y_{r I}^{u}+\sum_{j=1, j \neq p}^{n} \lambda_{j} y_{r j}^{u} \geq \mu y_{r I}+\sum_{j=1, j \neq p}^{n} \lambda_{j} y_{r j} \geq\left(1-\lambda_{p}\right) y_{r p} \geq\left(1-\lambda_{p}\right) y_{r p}^{l}$ $\mu x_{i I}^{l}+\sum_{j=1, j \neq p}^{n} \lambda_{j} x_{i j}^{l} \leq \mu x_{i I}+\sum_{j=1, j \neq p}^{n} \lambda_{j} x_{i j} \leq\left(\theta-\lambda_{p}\right) x_{i p} \leq\left(\theta-\lambda_{p}\right) x_{i p}^{u}$

Then $(\theta, \lambda, \mu)$ is a feasible solution to model (15) and since $e_{p}^{l}$ is optimal solution we have $e_{p}^{l} \leq e_{p}$.

Now let $\left(\theta^{\prime \prime}, \lambda^{\prime \prime}, \mu^{\prime \prime}\right)$ be an optimal solution of model (16), similarly is proved that $\mu^{\prime \prime} \geq 0$ and $\left(1-\lambda_{p}^{\prime \prime}\right) \geq 0$ and $\left(\theta^{\prime \prime}-\lambda_{p}^{\prime \prime}\right) \geq 0$. Then:

$\mu^{\prime \prime} y_{r I}+\sum_{j=1, j \neq p}^{n} \lambda_{j}^{\prime \prime} y_{r j} \geq \mu^{\prime \prime} y_{r I}^{l}+\sum_{j=1, j \neq p}^{n} \lambda_{j}^{\prime \prime} y_{r j}^{l} \geq\left(1-\lambda_{p}^{\prime \prime}\right) y_{r p}^{u} \geq\left(1-\lambda_{p}^{\prime \prime}\right) y_{r p}$ $\mu^{\prime \prime} x_{i I}+\sum_{j=1, j \neq p}^{n} \lambda_{j}^{\prime \prime} x_{i j} \leq \mu^{\prime \prime} x_{i I}^{u}+\sum_{j=1, j \neq p}^{n} \lambda_{j}^{\prime \prime} x_{i j}^{u} \leq\left(\theta^{\prime \prime}-\lambda_{p}^{\prime \prime}\right) x_{i p}^{l} \leq\left(\theta^{\prime \prime}-\lambda_{p}^{\prime \prime}\right) x_{i p}$ Thus $\left(\theta^{\prime \prime}, \lambda^{\prime \prime}, \mu^{\prime \prime}\right)$ is a feasible solution of model (14), hence $e_{p} \leq e_{p}^{u}$. There fore 
we have $e_{p}^{l} \leq e_{p} \leq e_{p}^{u}$, and the proof is completed.

\subsection{Integer programming model for bid selection with interval data}

The following pair of IP models have been constructed to select an optimal set of bids for models (7) and (8) or (12) and (13).

$$
\begin{aligned}
& \min \quad z_{0}=\sum_{j=1}^{n} z_{j} \\
& \min z_{0}=\sum_{j=1}^{n} z_{j} \\
& \text { s.t. } \quad \sum_{j=1}^{n} e_{j}^{l} z_{j}-e_{\text {pavg }}^{l} \sum_{j=1}^{n} z_{j} \geq 0 \text {, } \\
& \sum_{j=1}^{n} q_{j}=D \text {, } \\
& \text { s.t. } \quad \sum_{j=1}^{n} e_{j}^{u} z_{j}-e_{\text {pavg }}^{u} \sum_{j=1}^{n} z_{j} \geq 0 \text {, } \\
& q_{j}-c_{\text {jmax }} z_{j} \leq 0, \quad j=1, \ldots, n, \\
& \sum_{j=1}^{n} q_{j}=D \text {, } \\
& q_{j}-o_{j m i n} z_{j} \geq 0, \quad j=1, \ldots, n, \\
& q_{j}-c_{\text {jmax }} z_{j} \leq 0, \quad j=1, \ldots, n, \\
& q_{j} \geq 0 \\
& j=1, \ldots, n, \\
& q_{j}-o_{j \min } z_{j} \geq 0, \quad j=1, \ldots, n, \\
& z_{j} \in\{0,1\} \quad j=1, \ldots, n . \\
& q_{j} \geq 0 \\
& j=1, \ldots, n, \\
& z_{j} \in\{0,1\} \\
& j=1, \ldots, n \text {. }
\end{aligned}
$$

With comparison of the solutions, if $z_{j}^{*}$ in this models have a constant value, in final optimal solution $z_{j}^{*}$ 's value is constant. But if for some vendors $z_{j}^{*}$ 's value is variable, in final solution we defined $z_{j}^{*}$ as $z_{j}^{*} \in\{0,1\}$, where in this case buyer should select the vendor, itself and model isn't definitely determined.

\section{Application example}

We now apply this approach to a byer of food's products (for one goods) in Iran. There are 4 vendors in this district. Product price, quality and service time are considered to be the three most important factors in evaluating vendors. Price is utilized as the input, and quality and service time are considered as outputs.

In table 1 the interval input and interval outputs for these $D M U_{s}$ are given.

Table 1: The set of interval input and outputs

\begin{tabular}{|c|c|c|c|c|c|c|c|c|}
\hline$D M U_{j}$ & $x_{j}^{l}$ & $x_{j}^{u}$ & $y_{1 j}^{l}$ & $y_{1 j}^{u}$ & $y_{2 j}^{l}$ & $y_{2 j}^{u}$ & MOR & $C$ \\
\hline 1 & 19250 & 19250 & 1 & 1 & 0.7 & 1 & 500 & 4000 \\
2 & 18000 & 18000 & 2 & 2 & 0.2 & 0.8 & 500 & 4000 \\
3 & 22000 & 22000 & 1 & 1 & 0.7 & 1 & 500 & 4000 \\
4 & 14000 & 14450 & 7 & 7 & 0 & 0.3 & 500 & 4000 \\
\hline
\end{tabular}

MOR: minimum order requirement, C: capacity repressed.

The result of case (3) analysis are shown in table 2. 


Table 2: Vendors efficiencies
\begin{tabular}{|c|c|c|}
\hline$D M U_{j}$ & $e_{p}^{l}$ & $e_{p}^{u}$ \\
\hline 1 & 0.51 & 1.00 \\
2 & 0.22 & 0.92 \\
3 & 0.45 & 0.94 \\
4 & 0.97 & 1.00 \\
\hline
\end{tabular}

The corresponding input and output weights are shown in table 3.

Table 3: Output weights in lower and upper bound of efficiencies

\begin{tabular}{|c|c|c|c|c|}
\hline$D M U_{j}$ & $a_{1}^{l}$ & $a_{1}^{u}$ & $a_{2}^{l}$ & $a_{2}^{u}$ \\
\hline 1 & 0.000000 & 154.761905 & $1.40000 \mathrm{E}+4$ & $1.90952 \mathrm{E}+4$ \\
2 & $2.00000 \mathrm{E}+3$ & 0.000000 & 0.000000 & $2.06429 \mathrm{E}+4$ \\
3 & 0.000000 & 0.000000 & $1.40000 \mathrm{E}+4$ & $2.06429 \mathrm{E}+4$ \\
4 & $2.00000 \mathrm{E}+3$ & $1.95179 \mathrm{E}+3$ & 0.000000 & $1.12500 \mathrm{E}+3$ \\
\hline
\end{tabular}

It is evident from these result that vendor 4 is the best performer.

The demand for the buyer is $1500 \mathrm{~kg}$. We utilize the efficiency scores obtained in a 0 - 1 integer programming models shown in expressions (19) and (20). The results are shown in Table 4 and 5.

Table 4: Vendor selection and order quantities for model 7
\begin{tabular}{|c|c|c|}
\hline vendor & Selection & Order quantity \\
\hline 1 & 0 & 0 \\
2 & 0 & 0 \\
3 & 0 & 0 \\
4 & 1 & 1500 \\
\hline
\end{tabular}

\begin{tabular}{|c|c|c|}
\hline vendor & Selection & Order quantity \\
\hline 1 & 1 & 1500 \\
\hline 2 & 0 & 0 \\
\hline 3 & 0 & 0 \\
\hline 4 & 0 & 0 \\
\hline
\end{tabular}

Then $Z=(0,0,0,1)$ or $Z=(1,0,0,0)$ and $Q=(0,0,0,1500)$ or $Q=$ $(1500,0,0,0)$. In the present case scenario, one vendor needs to be selected because of the demand requirements. 


\section{Conclusions}

In this paper, we have reviewed models of Talluri (2002) and Zhu (2004). Some theorems about this models are proved. We have developed a new pair of models for dealing with interval data in every case. By solving these models an interval will be defined for the efficiency score. The model evaluations are integrated into a new pair of 0 - 1 integer programming formulation in determining the optimal set of vendors to be selected in meeting the demand requirements of the buyer without violating the minimum order necessities of the vendors. If case (2) and case (4) have interval input and output, for finding each vendor's interval efficiency can solve one problem that optimizes the sum of all of the efficiencies in their lower and upper bounds. One can prevent a big difference between the weights by applying weight control on models made in this paper.

ACKNOWLEDGMENTS. We do appreciate Mr Emil Minchev that reviewed the article in a short time and give us fruitful suggestions and recommendation and also the entire DEA group at science and research university.

\section{References}

[1] Charnes, A., Cooper, W. W., Rhodes, E., 1978. Measuring the efficiency of decision making units. European Journal of Operational Research 2, $429-444$.

[2] Dickson, G., 1966. An analysis of vendor selection systems and decisions. Journal of Purchasing 2, 28-41.

[3] Talluri, s., 2002. A buyer - seller game model for selection and negotiation of purchasing bids. European Journal of Operational Research 143 (1), 171 $-180$.

[4] Vijayan, J., 2000. Vendors offer tools to sell customized parts. Computerworld 34 (15), 73.

[5] Weber, C.A., Current, J.R., Desai, A., 1998. Non-cooperative negotiation strategies for vendor selection. European Journal of Operational Research 108 (1), 208-223.

[6] Weber, C.A., Desai, A., 1996. Determination of path to vendor market efficiency using parallel coordinates representation: A negotiation tool for buyers. European Journal of Operational Research 90 (1), 142-155. 
[7] Willis, T.H., Huston, C.R., Pohlkamp, F., 1993. Evaluation measures of just-in-time supplier performance. Production and Inventory Management Journal 34 (2), 1-6.

[8] Wise, R., Morrison, D., 2000. Beyond the exchange: The future of B2B. Harvard Business Review 78 (6), 86-96.

[9] Zhu, J., 2004. A buyer - seller game model for selection and negotiation of purchasing bids: Extensions and new models. European Journal of Operational Research 154 (1) 150 - 156.

Received: July 30, 2005 